\title{
Efeito da utilização de assistência técnica sobre a renda de produtores familiares do Brasil no ano de 2014
}

\author{
Impact evaluation of technical assistance on the income of Brazilian family \\ farmers in 2014
}

\author{
Adauto Brasilino Rocha Junior ${ }^{1}$ (D), Raniella Orquiza da Silva ${ }^{2}$ (D), Waldemiro Peterle Neto ${ }^{3}$ (D), \\ Cristiana Tristão Rodrigues ${ }^{4}$ (1)
}

${ }^{1}$ Escola Superior de Agricultura “Luiz de Queiroz” (ESALQ), Universidade de São Paulo (USP), Piracicaba (SP), Brasil. E-mails: adautojunior@usp.br; adauto.junior.20102@gmail.com

2Departamento de Economia, Universidade Federal de Viçosa (UFV), Viçosa (MG), Brasil. E-mail: raniella.silva@ufv.br

${ }^{3}$ Haskell Cosmética Natural, Viçosa (MG), Brasil. E-mail: waldemiro.neto@ufv.br

${ }^{4}$ Departamento de Economia, Universidade Federal de Viçosa (UFV), Viçosa (MG), Brasil. E-mail: cristiana.rodrigues@ufv.br

\begin{abstract}
Como citar: Rocha Junior, A. B., Silva, R. O., Peterle Neto, W., \& Rodrigues, C. T. (2020). Efeito da utilização de assistência técnica sobre a renda de produtores familiares do Brasil no ano de 2014. Revista de Economia e Sociologia Rural, 58(2), e194371. https://doi.org/10.1590/1806-9479.2020.19437
\end{abstract}

Resumo: Análises quantitativas dos efeitos da assistência técnica e extensão rural (ATER) são fundamentais para o aperfeiçoamento da política. Com o objetivo de avaliar o efeito da utilização de assistência técnica sobre a renda mensal dos agricultores familiares brasileiros, no presente trabalho aplicou-se o instrumento econométrico Propensity Score Matching, utilizando-se dados da Pesquisa Nacional por Amostras Domiciliares (PNAD) do ano de 2014. Os resultados obtidos evidenciaram acréscimo estatisticamente significativo de $\mathrm{R} \$ 490,54$ sobre a renda mensal dos agricultores familiares devido à utilização de assistência, o que comprova a efetividade da ATER enquanto instrumento de geração de renda. Além disso, a análise em nível agregado permite inferir que o acréscimo de renda mensal total para os agricultores familiares em 2014, devido às ações de ATER, foi estatisticamente superior ao gasto anual do governo com o Programa de Assistência Técnica e Extensão Rural na Agricultura Familiar. Conclui-se, desse modo, que há forte indício da viabilidade da expansão das ações de ATER, principalmente considerando que apenas 17\% dos agricultores familiares foram assistidos por tais serviços em 2014. A expansão da política, associada às análises sistêmicas para seu constante aperfeiçoamento, é, portanto, um caminho promissor para o desenvolvimento econômico da agricultura familiar no Brasil.

Palavras-chave: política agrícola, agricultura familiar, assistência técnica, capacitação.

Abstract: Quantitative analyzes of the effects of technical assistance and rural extension (Ater) are fundamental for the improvement of the policy. With the objective of evaluating the effect of the use of technical assistance on the monthly income of Brazilian family farmers, the econometric instrument Propensity Score Matching was applied in the present work, using data from the National Survey for Household Samples (PNAD) of the year 2014. The results evidenced a statistically significant increase of $\mathrm{R} \$ 490.54$ on the monthly income of the family farmers due to the use of assistance. In addition, the aggregate-level analysis allowedto infer that the increase in total monthly income for family farmers in 2014, due to Ater's actions, that was statistically higher than the government's annual expenditure with the Technical Assistance and Rural Extension Program in Family Agricultur . It is concluded that there is a strong indication of the viability of the expansion of Ater's actions, especially considering that only $17 \%$ of family farmers were assisted by such services in 2014. The expansion of the policy, coupled with systemic analysis for its constant improvement is, therefore, a promising route for the economic development of family agriculture in Brazil.

Keywords: Agricultural policy, family farming, technical assistance, training. 


\section{Introdução}

O setor primário desempenha papel fundamental na dinamização da economia brasileira. Verifica-se a importância desse setor ao considerar que, mesmo o Brasil ainda apresentando resquícios da recessão doméstica ${ }^{1}$, o crescimento de $1 \%$ do Produto Interno Bruto do país em 2017 adveio principalmente do desempenho da agropecuária (Brasil, 2018). Dessa maneira, o papel da agropecuária foi primordial para o - mesmo que modesto progresso do país, ao impactar na inflação e gerar um saldo recorde na balança comercial (Brasil, 2018).

O bom desempenho da agropecuária deve-se em grande parte à agricultura familiar, dado que essa categoria de produtores foi responsável por $38 \%$ da produção agrícola nacional em 2015, contribuindo com o fornecimento de produtos, como arroz, feijão, milho e carnes, itens básicos da cesta de alimentos brasileira (Castro, 2015). No entanto, embora apresente potencial econômico e social considerável, a agricultura familiar enfrenta dificuldades próprias, características de uma atividade que é, segundo (Batalha et al., 2005), exercida por produtores pouco qualificados, inseridos em um ambiente altamente competitivo e tecnificado.

Segundo Abramovay (1998), há inúmeras restrições ao desenvolvimento da agricultura familiar no Brasil, como a dificuldade na construção de capital social e na inserção nos mercados, fatores esses que impedem que os agricultores familiares valorizem os atributos de sua localização, construam mercados e transformem, a seu favor, o ambiente institucional no qual estão inseridos. Dessa forma, políticas de acesso ao crédito rural e à provisão pública da assistência técnica e extensão rural (ATER) são de fundamental importância para o desenvolvimento da agricultura familiar e a segurança alimentar do país.

Desde os anos 1950, o serviço de assistência técnica rural é institucionalizado, tendo em vista a criação das associações de crédito e assistência rural nos estados. Essas associações elaboravam projetos técnicos com a finalidade de obtenção de crédito pelos produtores junto a instituições financeiras (Peixoto, 2008). No entanto, ao apresentarem uma concepção conservadora de desenvolvimento, tais ações de extensão rural privilegiaram os agricultores empresariais (Dias, 2008), preterindo os produtores familiares, colocando-os à margem de benefícios, como o seguro da produção e o crédito rural (Hampf, 2013), o que culminou na elevação da desigualdade no campo.

A Constituição de 1988 e, paralelamente, o movimento sindical rural auxiliaram para que políticas públicas fossem direcionadas para a redução dessa desigualdade (Hampf, 2013). Foi nesse contexto que, em 1996, instituiu-se o Programa Nacional de Fortalecimento da Agricultura Familiar (PRONAF) ${ }^{2}$, tendo como principais objetivos o acesso ao crédito e o incentivo à assistência técnica para os agricultores de base familiar (Dias, 2008). No entanto, o impacto do PRONAF sobre variáveis, como renda, produtividade e emprego, não se apresenta de forma consensual na literatura. Enquanto alguns trabalhos empíricos demonstraram que o PRONAF alcançou, pelo menos parcialmente, os resultados esperados (Feijó, 2001; Damasceno et al., 2011), outras pesquisas não confirmaram o impacto do PRONAF sobre o nível de investimento, a produtividade e a renda (Kageyama, 2003; Pereira, 2004; Magalhães, 2005; Hampf, 2013).

A literatura ainda discursa, de forma mais abrangente e teórica, sobre o desempenho e atuação dos serviços de assistência técnica e extensão rural para a agricultura familiar (Silva, 2013). Batalha et al. (2005) ressaltam a importância do emprego de técnicas adequadas para uma maior competitividade dos agricultores familiares e Dias (2008) apresenta um panorama histórico das políticas públicas voltadas para extensão rural e suas mudanças institucionais. No entanto, devido à escassez de dados e às limitações metodológicas, nenhum dos trabalhos

\footnotetext{
${ }^{1} \mathrm{O}$ Instituto Brasileiro de Economia (2017) estabeleceu que a recessão se compreendeu no período de 2014 a 2016, sendo a mais longa em comparação às nove ocorridas desde 1980.

${ }^{2}$ Ressalta-se que o PRONAF é uma política de crédito rural. No entanto, está intimamente relacionado à assistência técnica, tendo em vista que o programa pronunciava o pagamento à assistência técnica aos projetos de crédito rural (Dias, 2008).
} 
recentes analisou, em termos quantitativos, o efeito das ações de assistência técnica sobre variáveis relevantes, como a renda dos produtores.

Nesse contexto, o presente trabalho tem a finalidade de contribuir com o debate acadêmico e político da ATER, ao analisar o efeito da utilização dos serviços de assistência técnica sobre a renda dos agricultores familiares brasileiros, em 2014. Para tanto, utilizam-se dados da Pesquisa Nacional por Amostra de Domicílios (PNAD) de 2014 (Instituto Brasileiro de Geografia e Estatística, 2017), de maneira a - através da metodologia Propensity Score Matching - avaliar a hipótese de que os agricultores familiares que utilizaram o serviço de ATER usufruíram de acréscimos na renda, comparativamente ao grupo de agricultores familiares que não recorreram a tais serviços. Além disso, estimaram-se, para o ano de 2014: 1) o acréscimo total na renda mensal dos agricultores assistidos, decorrente da utilização de assistência técnica; 2) o acréscimo potencial de renda caso os agricultores não assistidos utilizassem os serviços de Ater, e 3) o acréscimo potencial total, caso todos os agricultores familiares do Brasil fossem assistidos. Com base nos resultados, discute-se a efetividade da ATER como instrumento político de geração de renda.

A análise de uma política caracterizada pela ação estatal com foco em uma categoria de produtores que, mesmo enfrentando muitos entraves na produção, desempenham notória contribuição para a segurança alimentar nacional é de grande contribuição na formulação de políticas públicas.

\section{A assistência técnica e a agricultura familiar}

\subsection{Aspectos teóricos da assistência técnica ao agricultor familiar}

No início do século $X X$, os Estados Unidos institucionalizaram, pela primeira vez na História, a ação de extensão rural. Desde então, são desenvolvidos estudos buscando relacionar esses serviços com o desenvolvimento rural. Duas teorias são amplamente discutidas no arcabouço teórico em torno do debate sobre a importância da assistência técnica para o desenvolvimento rural, a teoria da Agricultura de Altos Insumos e a teoria da Mudança Tecnológica Induzida. Ambas defendem a modernização da agricultura, desvinculando-a da concepção de agricultura tradicional.

A teoria da Agricultura de Altos Insumos, atribuída à Schultz (1965), define os agricultores como racionais e eficientes na obtenção de recursos, sendo a dificuldade de acesso a serviços técnicos e econômicos o principal entrave ao desenvolvimento rural. Segundo essa percepção, o acesso à capacitação seria um instrumento fundamental para o aumento da produtividade nos empreendimentos agrícolas. Ruttan \& Hayami (1984), a partir da teoria de Mudança Tecnológica Induzida, complementam essa discussão, destacando que o progresso técnico é responsável pela substituição de fatores de produção escassos por fatores abundantes. Há, portanto, nessa última concepção, forte ênfase na identificação e difusão da tecnologia como caminho para o desenvolvimento rural (Gomes et al., 2016).

Ao discutirem a importância da capacitação técnica para o desenvolvimento rural, essas teorias salientam o termo extensão rural como fator crítico para o progresso econômico da agricultura familiar. O termo extensão rural envolve grande complexidade ao poder ser conceituado de três diferentes formas: como processo, como instituição e como política. A primeira forma remete ao sentido literal, ou seja, o processo de transmissão do conhecimento, da fonte geradora ao público rural (receptor final). Já a percepção da extensão rural como instituição - adotando o conceito Schumpteriano de instituições enquanto órgãos - concerne às organizações estatais que prestam serviço de assistência técnica e extensão rural. Por último, referindo-se ao conceito de política pública, tem-se os serviços de ATER, definidos como políticas traçadas pelas três esferas do governo (federal, estadual e municipal), podendo ser executadas por organizações privadas e/ou públicas (Peixoto, 2008). Nesse ínterim, tem-se que o termo assistência técnica e extensão rural (ATER) mais vinculado à problemática do presente estudo está relacionado ao terceiro conceito, sendo o serviço de ATER compreendido como uma política pública. 
A abordagem dessa política com foco em seu efeito sobre a agricultura familiar é de grande relevância, considerando que há um intenso debate ideológico a respeito da função econômica e social da agricultura familiar no país. É importante ressaltar que o termo agricultura familiar se popularizou no Brasil, de acordo com Wanderley (2013), depois da implantação do Programa Nacional de Fortalecimento da Agricultura Familiar (Pronaf) em 1995, cujo objetivo principal foi o de fomentar o desenvolvimento sustentável da agricultura familiar. Nas resoluções da Lei n. ${ }^{\circ} 11.326 / 2006$ e nas atualizações dadas pelo Decreto N. ${ }^{\circ}$ 9.064/2017, agricultor familiar é o empreendedor familiar rural que pratica atividades no meio rural, possui área de até quatro módulos fiscais, mão de obra predominantemente familiar, bem como a renda e o gerenciamento do empreendimento são vinculados à própria família (Brasil, 2017). No entanto, a abrangência do conceito é comumente discutida entre legisladores e especialistas. Enquanto, para alguns, se confunde com a definição operacional adotada pelo Pronaf para definir os beneficiários do programa, para outros, a categoria corresponde a uma camada de agricultores capazes de se adequar às exigências modernas do mercado, opondo-se à ideia de pequenos produtores, que seriam incapazes de assimilar essas inovações (Wanderley, 2013).

Castro (2015) afirma que, apesar da importância histórica, a classe dos agricultores familiares recebeu pouca atenção do poder público ao longo do tempo, sendo por várias vezes negligenciada. Adicionalmente, Guilhoto et al. (2007) presumem que a sobrevivência deste setor produtivo se torna incerta, na medida em que o ramo é desorganizado e ineficaz para gerir seus próprios interesses. A dificuldade de gestão normalmente está associada à diversidade de sistemas e estratégias produtivas que determinam objetivos distintos, fazendo com que a força do setor seja dissipada em grupamentos locais (Guilhoto et al., 2007). No entanto, Buainain et al. (2003) mencionam que o produtor familiar, quando recebe apoio suficiente, é capaz de produzir uma renda total, incluindo a de autoconsumo, superior ao custo de oportunidade do trabalho, o que viabilizaria a atividade.

Segundo Batalha et al. (2005), há um consenso entre formuladores e gestores de políticas públicas de que a competitividade da agricultura familiar só pode ser alcançada por meio da adoção de práticas que estimulem a cooperação entre os agentes econômicos da cadeia produtiva, incluindo o governo. Ademais, o autor afirma que uma das maneiras de fortalecer a agricultura familiar é por meio da agregação de valor aos seus produtos. Essa ideia realça a importância de ações de capacitação como a assistência técnica e a extensão rural (ATER), que devem não apenas abordar os aspectos técnicos da atividade, mas também tratar das características gerenciais.

\subsection{Assistência técnica e agricultura familiar no Brasil}

A origem dos serviços de ATER no Brasil se situa em 1948, quando foi criada a Associação de Crédito e Assistência Rural (ACAR) no estado de Minas Gerais, sob recomendação do empresário norte-americano Nelson Rockfeller (Castro, 2015). Conforme Castro (2015), a ACAR foi estruturada de acordo com o modelo norte-americano de difusão de inovações, que basicamente atribuía à extensão rural a missão de oferecer assistência técnica e financeira aos produtores rurais para que adotassem as inovações desenvolvidas em institutos de pesquisa agrícola.

Segundo Oliveira (1999), ao final da década de 1950, os serviços de ATER estavam presentes na metade dos estados brasileiros, abrangendo completamente as regiões Sudeste e Sul, e expandindo-se para os estados do Nordeste (CE, PE, BA, RN, PB) e Centro-Oeste (GO). Caporal (2008) ressalta que, em 1956, a Associação Brasileira de Crédito e Assistência Rural (ABCAR) - entidade de caráter privado - congregou todas as ACARs segundo um modelo centralizado e vertical de orientação. Entretanto, com o crescimento dessas instituições, o Estado brasileiro gradativamente as trouxe para sua esfera de influência e controle, oferecendo apoio financeiro e exigindo em troca o apoio ao seu projeto de desenvolvimento rural, culminando, em 1975, com a criação da Empresa Brasileira de Assistência Técnica e Extensão Rural (Embrater), que incorporou a ABCAR, e alterou o nome das unidades estaduais de ACAR para empresa estadual de assistência técnica e extensão rural (Emater), as quais ficaram subordinadas ao seu controle (Castro, 2015). 
É importante ressaltar que, apesar da extensão das ações dos órgãos de Emater estaduais, existem outras entidades que oferecem o serviço de assistência aos produtores familiares, como algumas agências municipais, estaduais e federais (por exemplo, as secretarias municipais de agricultura), e até mesmo empresas privadas, como algumas compradoras da produção para beneficiamento ou vendedoras de insumos, as quais disponibilizam técnicos para atender aos agricultores no campo.

O último Censo Agropecuário, realizado em 2006 pelo Instituto Brasileiro de Geografia e Estatística (IBGE), trouxe informações relevantes sobre a abrangência da orientação técnica aos produtores. Um resultado observado na pesquisa foi que a orientação técnica é utilizada em $22 \%$ dos estabelecimentos rurais, os quais ocupam quase metade das terras utilizadas na agricultura. Além disso, a área média dos agricultores assistidos é de 228 hectares (ha), enquanto a dos não assistidos é de 42 ha. Tais números evidenciam uma clara segmentação da assistência técnica em função de sua origem e do tamanho das propriedades assistidas (Instituto Brasileiro de Geografia e Estatística, 2009).

Ainda segundo os dados do Censo Agropecuário de 2006, a assistência técnica originária do governo atingiu $43 \%$ dos estabelecimentos e está mais voltada para os estabelecimentos menores, com área média de 64 ha. Já a assistência de empresas privadas de planejamento atendeu às propriedades com área média de 506 ha (Instituto Brasileiro de Geografia e Estatística, 2009). O Censo também mostrou que o nível de escolaridade da pessoa responsável pela propriedade tem forte relação com o recebimento da assistência técnica. Dos produtores com instrução igual ou inferior ao Ensino Fundamental incompleto, apenas 16,8\% receberam assistência técnica; para os produtores com Ensino Fundamental completo, este percentual sobe para 31,7\%; e para os produtores com nível superior, excetuando-se aqueles com formação em ciências agrárias e veterinária, a assistência técnica alcança 44,7\% dos estabelecimentos (Instituto Brasileiro de Geografia e Estatística, 2009). Esse resultado é preocupante, pois apenas 19,6\% dos produtores entrevistados no censo possuíam Ensino Fundamental completo ou qualificação superior.

Os fatores regionais também apresentaram diferenças quando se trata de utilização de assistência técnica. Quirino et al. (2002), mostraram que o percentual de propriedades atendidas é de $50,5 \%$ das unidades produtivas no Sul, $41,5 \%$ no Sudeste, $32 \%$ no Centro-Oeste, $14,6 \%$ no Nordeste e $14,5 \%$ no Norte, o que evidencia um contraste entre os graus de abrangência da assistência técnica para as diferentes regiões.

Apesar da importância desses resultados, não existem análises objetivas do efeito da assistência técnica sobre a renda de agricultores familiares em nível nacional e a principal fonte de dados para esse tipo de estudo, que é o Censo Agropecuário, não é atualizada desde 2006. Por esse motivo, considerando a escassez de estudos com dados mais recentes sobre o tema, entende-se que o presente trabalho propicia uma relevante contribuição, não apenas à literatura sobre assistência técnica aos agricultores familiares brasileiros, mas também ao aperfeiçoamento da política de ATER no território nacional.

\section{Metodologia}

\subsection{O Propensity Score Matching como instrumento econométrico}

A estimação do efeito da assistência técnica sobre a renda dos agricultores familiares é desafiadora, uma vez que não existe uma base de dados contínua que permita a comparação entre a renda antes e após a utilização da assistência, para um mesmo agricultor, ao longo do tempo. Por esse motivo, um instrumento econométrico bastante conveniente é o Propensity Score Matching (PSM).

O princípio da aplicação desse método, extensamente discutido na literatura ${ }^{3}$, é considerar que existem dois grupos (ou indivíduos) que devem ser comparados, os quais se diferem apenas pelo fato de participar ou não de determinado tratamento, o qual, no

\footnotetext{
${ }^{3}$ Alguns dos principais autores que que estudaram a metodologia são Rosembaun \& Rubin (1983), Rosenbaum (2002), Rubin (1973, 1979), Heckman et al. (1998), Abadie et al. (2002), Lalonde (1986) e Dehejia \& Wahba (1999). Uma síntese didática da aplicação e dos detalhes teóricos da metodologia é realizada por Caliendo \& Kopeinig (2008).
} 
presente trabalho, é a utilização de assistência técnica. Menezes Filho et al. (2012) relatam que o ideal seria analisar o valor da variável dependente para o mesmo indivíduo, sendo que este estaria ao mesmo tempo na situação de não tratado e de tratado. Assim, considerando um tratamento binário, assumindo $\mathrm{Di}=1$ para o indivíduo $i$, que utilizou assistência técnica, e 0 , caso contrário, os efeitos na variável de interesse - renda mensal do empreendimento [Yi(D)] - poderiam ser definidos como:

$$
\tau_{i}=Y_{i}(1)-Y_{i}(0)
$$

Em que:

$\tau_{i}=$ efeito do tratamento sobre a renda do indivíduo i;

$Y_{i}(1)$ = renda do indivíduo i após o tratamento;

$Y_{i}(0)=$ renda do indivíduo i caso ele não recebesse o tratamento.

Nesse caso, o efeito médio do tratamento seria dado por:

$E(\tau \mid D=1)=E[Y i(1) \mid D=1]-E[Y i(0) \mid D=1]$

Como não é possível utilizar, de forma simultânea, o mesmo indivíduo como tratado e não tratado, e considerando que a seleção para o tratamento é não aleatória, a aplicação do PSM permite a identificação dos indivíduos mais semelhantes o possível de cada indivíduo existente na amostra, tendo como diferença apenas o fato de ter recebido ou não assistência técnica.

Para chegar a esse contrafactual, no PSM, realiza-se o emparelhamento a partir de uma série de características observáveis. Uma dificuldade que surge, no entanto, consiste na necessidade de se considerar o efeito de diversas covariáveis (variáveis que representam características dos indivíduos e que afetam simultaneamente a probabilidade de participação do tratamento e o produto potencial), para que os indivíduos possam ser pareados com seus semelhantes. Uma opção adotada, segundo os trabalhos de Rosenbaum \& Rubin (1983) e dos principais autores da área, é reunir a informação de todas as características do indivíduo em uma única variável, denominada como escore de propensão.

O método mais comum para o cálculo do escore de propensão é a regressão por meio de um modelo de escolha qualitativa (Logit ou Probit) da probabilidade de participação do tratamento como função das covariáveis. A ideia, nesse caso, é usar o valor da probabilidade de participação do tratamento para identificar indivíduos semelhantes.

Para que isso seja possível, duas hipóteses devem ser consideradas. Primeiramente, a hipótese de independência condicional (ou seleção nos observáveis) assume que todas as variáveis que influenciam o tratamento e o produto potencial, simultaneamente, estão incluídas no vetor de covariáveis. Matematicamente, tem-se:

$Y i(0), Y j(I) \amalg D \mid X, \forall X$

Em que:

Yi(0) e Yj(1) = renda mensal domiciliar dos indivíduos $i$ (não tratado) e $j$ (tratado) pareados;

$\mathrm{D}=$ dummie de tratamento;

$X=0$ vetor de covariáveis.

Com o objetivo de respeitar essa hipótese, incluíram-se no vetor de covariáveis todas as variáveis associadas à probabilidade de utilização de assistência técnica e ao resultado potencial da renda mensal do empreendimento, as quais são apresentadas no Quadro 1. 
Quadro 1- Covariáveis incluídas no modelo

\begin{tabular}{|c|c|}
\hline Variável & Descrição \\
\hline Masc & Variável dummy do sexo masculino \\
\hline Idade & Anos de idade \\
\hline Nbranco & Variável dummy que indica cor de pele não branca \\
\hline Estudo & Variável contínua de anos de estudo \\
\hline PRONAF & Variável dummy que indica a participação no PRONAF \\
\hline Compempresa & Variável dummy que indica empresa como principal compradora da produção \\
\hline Compcooperativa & Variável dummy que indica cooperativa como principal canal de comercialização \\
\hline Compintermediário & Variável dummy que indica intermediários como principais compradores \\
\hline Compproprietário & $\begin{array}{l}\text { Variável dummy que indica o proprietário do empreendimento agrícola como } \\
\text { principal canal de comercialização }\end{array}$ \\
\hline Compconsdireto & Variável dummy que indica venda direta ao consumidor final \\
\hline Outrocomprador & $\begin{array}{l}\text { Variável dummy que indica que outro comprador é o principal canal de } \\
\text { comercialização }\end{array}$ \\
\hline Região & Indica em qual das cinco regiões o indivíduo se encontra \\
\hline Cessionário & $\begin{array}{l}\text { Variável dummy que indica o indivíduo na condição de cessionário do } \\
\text { empreendimento }\end{array}$ \\
\hline Posseiro & $\begin{array}{l}\text { Variável dummy que indica o indivíduo na condição de posseiro do } \\
\text { empreendimento }\end{array}$ \\
\hline Proprietário & $\begin{array}{l}\text { Variável dummy que indica o indivíduo na condição de proprietário do } \\
\text { empreendimento }\end{array}$ \\
\hline Parceiro & $\begin{array}{l}\text { Variável dummy que indica o indivíduo na condição de parceiro do } \\
\text { empreendimento }\end{array}$ \\
\hline OCond & $\begin{array}{l}\text { Variável dummy que indica o indivíduo em outra condição em relação ao } \\
\text { empreendimento }\end{array}$ \\
\hline ProdSubsist & $\begin{array}{l}\text { Variável dummy que indica que mais da metade da alimentação domiciliar foi } \\
\text { retirada da produção do empreendimento }\end{array}$ \\
\hline EmpreTemp & Variável dummy para indivíduo que exerceu emprego temporário \\
\hline EmprePerm & Variável dummy para indivíduo que exerceu permanente \\
\hline
\end{tabular}

Fonte: Elaboração própria.

É importante considerar que a variável área do empreendimento não foi incluída devido às limitações da base de dados. No entanto, as variáveis dummy de principal comprador da produção, existência de empregados temporários e permanentes, e finalidade da produção, por serem potencialmente correlacionadas com a área do empreendimento, eliminam grande parte do possível viés decorrente da omissão dessa variável.

A hipótese do suporte comum (ou sobreposição), por sua vez, exclui a possibilidade de previsibilidade perfeita da ocorrência do tratamento. Isso significa que tanto os indivíduos tratados quanto os não tratados apresentam uma probabilidade positiva de serem tratados, e uma probabilidade positiva de não serem tratados. Matematicamente:

$0<P(D=l \mid X)<1$

Em que $P(D=l \mid X)$ é a probabilidade de participação do tratamento dado o conjunto de covariáveis $X$. A importância dessa hipótese é garantir que indivíduos tratados e não tratados possam apresentar escores de propensão similares, o que é condição fundamental para que o pareamento seja possível.

Os diferentes métodos de utilização do escore de propensão para comparar tratados e não tratados caracterizam diferentes técnicas de pareamento. Segundo Caliendo \& Kopeinig (2008), a técnica mais objetiva e a mais utilizada em trabalhos que aplicam o PSM é o pareamento pelo 
vizinho mais próximo (NN, do inglês Nearest Neighbour). No NN, utiliza-se como contrafactual a média da variável sobre a qual objetiva-se avaliar o impacto do programa (no presente caso, tratase da renda per capita do agricultor familiar) para os $n$ indivíduos com os escores de propensão mais próximos do valor do indivíduo utilizado como base.

Segundo Caliendo \& Kopeinig (2008), a especificação do NN envolve dois trade-offs entre viés e variância, um na escolha do processo (com reposição ou sem reposição) e outro na escolha do número de vizinhos. Enquanto o processo com reposição permite que determinada observação possa ser usada como contrafactual para mais de um indivíduo, no processo sem reposição, os indivíduos podem ser utilizados uma única vez. Desse modo, com reposição, a qualidade média do pareamento é maior e diminui-se o viés na estimação do efeito de tratamento, o que é útil quando a distribuição dos escores é bastante diferente entre tratados e não tratados (Caliendo \& Kopeinig, 2008), uma característica da amostra utilizada no presente trabalho. No entanto, isso reduz o número de não participantes distintos usados para construir o resultado contrafactual e, assim, aumenta a variância do estimador (Smith \& Todd, 2005). Com relação ao número de vizinhos utilizados, quando se utiliza um número maior destes, a variância do estimador do efeito do tratamento diminui; no entanto, o viés aumenta, pois indivíduos menos semelhantes serão comparados entre si (Caliendo \& Kopeinig, 2008). Considerando que a amostra utilizada no presente trabalho é relativamente grande e priorizando-se a minimização do possível viés na estimação, adotou-se o pareamento utilizando-se apenas um vizinho mais próximo, e com reposição.

Após o pareamento dos indivíduos, é possível estimar o efeito do tratamento. No presente trabalho, o efeito é dado pela diferença entre a renda mensal domiciliar do indivíduo base e a renda mensal domiciliar do seu contrafactual (o vizinho mais próximo). Desse modo, obtém-se o efeito da assistência sobre a renda para cada indivíduo da amostra (tratado ou não tratado). Com base nos efeitos individuais, é possível estimar o efeito médio do tratamento (ATE), por meio da média do efeito estimado para todos os indivíduos da amostra, e o efeito médio do tratamento sobre os tratados (ATT), por meio da média do efeito estimado para os indivíduos que receberam tratamento. Além disso, é possível agregar os efeitos individuais do tratamento e estimar o acréscimo total de renda que a assistência técnica gerou, no ano de 2014, e o acréscimo de renda potencial, caso todos os agricultores recebessem assistência técnica.

A discussão desenvolvida no presente trabalho baseia-se nos resultados obtidos para o ATE, o ATT e os acréscimos de renda estimados em nível agregado. Com base nos resultados obtidos, discute-se a viabilidade econômica da prestação de serviços de ATER pelo governo.

\subsection{Fonte e tratamento dos dados}

Utilizou-se, como banco de dados, a Pesquisa Nacional por Amostra de Domicílios (PNAD) de 2014, porque, neste ano, a PNAD disponibilizou, na forma de pesquisa suplementar, um levantamento de dados sobre Acesso à Internet e à Televisão, e Inclusão Produtiva, sendo que, entre as informações, existem variáveis relacionadas à atividade agrícola, como o fato de ter recebido assistência técnica no último trabalho, a utilização do Pronaf, o principal comprador da produção, entre outras.

É importante ressaltar que, pelo fato de não existir na PNAD uma classificação que permita a identificação direta dos agricultores familiares - um perfil específico de produtor rural -, foi realizado o recorte amostral para que restassem, na amostra, apenas observações referentes à agricultores familiares. Por esse motivo, foram excluídos da amostra: os indivíduos residentes na zona urbana; aqueles que não se encontravam em condição de pessoa de referência da família; aqueles com renda anual bruta maior que 360 mil reais, e as observações referentes aos empreendimentos com área maior que 480 hectares $^{4}$. Devido à dificuldade em realizar a exclusão das observações referentes aos empreendimentos com área maior que quatro módulos fiscais

\footnotetext{
${ }^{4}$ A Lei N. ${ }^{\circ} 11.326$, de 24 de julho de 2006, define, como uma das características do agricultor familiar, que o empreendimento deveria possuir área de até quatro módulos fiscais. No entanto, o módulo fiscal é uma unidade de medida agrária instituída pela Lei n. ${ }^{\circ}$ 6.746, de 10 de dezembro de 1979, que representa a área mínima necessária para as propriedades rurais serem consideradas economicamente viáveis (Landau et al., 2012). Essa área varia conforme o município, podendo medir de 5 a 120 hectares.
} 
para cada município, considerando que a menor unidade territorial de agregação que a PNAD permite é em nível de estados ou regiões metropolitanas, foi realizada a dropagem (exclusão) das observações para as quais a área do empreendimento é maior do que 480 hectares $^{5}$, o que permitiu a eliminação de alguns outliers.

Apesar de a preparação dos dados ser realizada com o objetivo de delimitar a amostra para a população mais próxima o possível daquela definida como agricultores familiares, existem limitações decorrentes de aspectos da base de dados. A principal limitação encontrada é o pouco número de observações com que foi informada a área do empreendimento. Isso é compreensível devido à dificuldade dos agricultores em informar a área de seu empreendimento no exato momento da coleta de dados.

Porém, considerando a relevância do tema e a rica disponibilidade de outras variáveis explicativas, entende-se que a existência de algumas limitações não inviabiliza a análise. Em vez disso, o desafio de trabalhar com as informações existentes, minimizando a perda resultante das deficiências da base de dados e extraindo o máximo possível de informações úteis, torna-se mais um diferencial do presente estudo.

A consideração de que os dados da PNAD são oriundos de um processo de amostragem complexa que abrange todo o território nacional é de grande relevância para a confiabilidade dos resultados obtidos. Esse tipo de amostragem caracteriza-se pela não aleatoriedade na escolha das observações e, por esse motivo, exige procedimentos específicos de preparação de dados, mais especificamente a atribuição dos pesos amostrais às observações, para que os estimadores obtidos a partir de sua análise não sejam viesados. Esse procedimento foi realizado na presente pesquisa nas fases de estimação do propensity score, estimação dos efeitos do tratamento e estimação dos acréscimos de renda no nível agregado.

\section{Resultados e discussão}

A confiabilidade dos resultados obtidos pela metodologia do Propensity Score Matching depende, basicamente, da qualidade do pareamento realizado, uma vez que é nessa etapa que são definidos quais indivíduos serão comparados para identificar o efeito da política. Por esse motivo, no presente trabalho foram testados os pareamentos por meio dos modelos Logit e Probit, visando avaliar qual dos dois propiciou maior redução do viés médio entre as variáveis que caracterizam os indivíduos. Na Tabela 1, são apresentados os vieses característicos da amostra antes e após o pareamento com Logit e Probit.

Tabela 1 - Significância das regressões logísticas e média e mediana do viés antes e após o pareamento

\begin{tabular}{cccccc} 
Amostra & Ps R2 & LR chi2 & p>chi2 & MeanBias & MedBias \\
Probit antes do pareamento & 0,251 & 836,51 & 0,000 & 24,1 & 15,2 \\
Pareamento por Probit & 0,011 & 18,54 & 0,776 & 4,3 & 3,5 \\
Pareamento por Logit & 0,008 & 13,21 & 0,963 & 3,3 & 2,4 \\
\hline
\end{tabular}

Fonte: Elaboração própria com base no modelo estimado.

É possível observar na Tabela 1 que o viés médio diminui de forma substancial após o pareamento, tanto para o Probit quanto para o Logit, um indicativo de boa qualidade do procedimento. Além disso, o pseudo $\mathrm{R}^{2}$ também é reduzido e ambas as regressões deixam de ser significativas, demonstrando que as características dos indivíduos pareados não permite a previsão da utilização ou não da assistência técnica, o que evidencia que os modelos Logit e Probit foram adequadamente especificados (Smith \& Todd, 2005), e que os grupos pareados são consistentemente comparáveis (Blundell et al., 2005; Sianesi, 2004).

\footnotetext{
${ }^{5}$ Destaca-se, no entanto, na análise estatística das áreas do empreendimento informadas na amostra, que 98,92\% delas possuem 19,36 hectares ou menos, obedecendo ao requisito de acesso quando o módulo fiscal é o menor possível (5 ha). Conclui-se, portanto, que, considerando a estrutura fundiária verificada na amostra, na qual predominam as pequenas propriedades com tamanho abaixo de quatro módulos fiscais (em seu valor mínimo verificado no Brasil), o prejuízo decorrente da impossibilidade de truncar a amostra perfeitamente com relação à área máxima do empreendimento é praticamente irrelevante.
} 
Observando-se os valores das médias e medianas do viés na Tabela 1, é possível inferir que o Logit oferece o melhor pareamento, resultado também perceptível pelo menor valor do pseudo $\mathrm{R}^{2}$ e do $\mathrm{p}$-valor da regressão pós-pareamento. Definida a escolha do Logit para o pareamento, $\mathrm{o}$ próximo passo é avaliar o ajuste do modelo estimado para explicar a probabilidade de recebimento de assistência técnica. Na Tabela 2, são apresentadas as estatísticas da estimação.

Os resultados apresentados na Tabela 2 evidenciam que 16 das 24 variáveis explicativas apresentaram efeito estatisticamente significativo, no nível de 10\% de significância, sobre a probabilidade de utilização de assistência técnica pelos agricultores familiares brasileiros. Esse resultado é conveniente, pois, na escolha das variáveis, consideraram-se todos os dados observáveis disponíveis na PNAD de 2014 que potencialmente influenciaram os indivíduos na decisão pela utilização da assistência e que tais variáveis são simultaneamente correlacionadas com a renda do empreendimento, em conformidade com a recomendação de Menezes Filho et al. (2012). Desse modo, o bom ajuste do modelo é um indício da boa capacidade em estimar a probabilidade de utilização da assistência técnica por agricultores familiares - que equivale ao escore de propensão na presente análise - com base nas características representadas pelas variáveis explicativas, propiciando o pareamento condizente com a hipótese de seleção dos observáveis.

Tabela 2 - Resultados da estimação do modelo Logit para a utilização de assistência técnica em 2014

\begin{tabular}{cccc} 
Variáveis & Coeficientes & Erros-padrão & p-valor \\
\hline Idade & $-0,0002599^{\tilde{n}}$ & 0,0044866 & 0,95 \\
utilPRONAF & $1,6137370^{1 \%}$ & 0,1290701 & 0,00 \\
Estudo & $0,0947194^{1 \%}$ & 0,0150961 & 0,00 \\
FEM & $0,0334553^{\tilde{n}}$ & 0,2075865 & 0,87 \\
Nbranco & $-0,1968083^{\tilde{n}}$ & 0,1253643 & 0,12 \\
Cessionário & $-0,7558436^{1 \%}$ & 0,3010991 & 0,01 \\
Posseiro & $0,6023188^{5 \%}$ & 0,2793538 & 0,03 \\
Arrendatário & $-0,0991067^{\tilde{n}}$ & 0,2828275 & 0,73 \\
Parceiro & $-0,2250188^{\tilde{n}}$ & 0,2485131 & 0,37 \\
Outracondicao & $-1,2282990^{\tilde{n}}$ & 0,7590997 & 0,11 \\
Prodsubsist & $-0,4814601^{1 \%}$ & 0,1830201 & 0,01 \\
Naoconsome & $-0,3257373^{1 \%}$ & 0,1266033 & 0,01 \\
Temempregtemp & $1,1523860^{1 \%}$ & 0,2634948 & 0,00 \\
Temempregperm & $0,4319194^{10 \%}$ & 0,2523020 & 0,09 \\
Compconsdireto & $-0,6345547^{1 \%}$ & 0,1933411 & 0,00 \\
Compempresa & $0,8271824^{1 \%}$ & 0,1542634 & 0,00 \\
Compcooperativa & $0,3651574^{10 \%}$ & 0,2050591 & 0,08 \\
Ccompproprietário & $1,1270130^{10 \%}$ & 0,5931916 & 0,06 \\
Ccompintermediário & $-0,0571109^{\tilde{n}}$ & 0,1657485 & 0,73 \\
Outrocomprador & $-0,3858953^{\tilde{n}}$ & 0,8012840 & 0,63 \\
Norte & $-1,2714450^{1 \%}$ & 0,1721670 & 0,00 \\
Nordeste & $-1,5148930^{1 \%}$ & 0,1786778 & 0,00 \\
CO & $-1,6271430^{1 \%}$ & 0,2390737 & 0,00 \\
Sudeste & $-1,0074600^{1 \%}$ & 0,1596845 & 0,00 \\
_cons & $-1,3655670^{1 \%}$ & 0,3217976 & 00 \\
\hline
\end{tabular}

Fonte: Elaboração própria com base no modelo estimado. ${ }^{1 \%}$ Coeficiente estatisticamente significativo a $1 \%$ de significância. ${ }^{5 \%}$ Coeficiente estatisticamente significativo a 5\% de significância. ${ }^{10 \%}$ Coeficiente estatisticamente significativo a 10\% de significância. ${ }^{\tilde{n}}$ Coeficiente estatisticamente não significativo a 10\% de significância.

É importante ressaltar que, segundo Menezes Filho et al. (2012) a utilização e a significância das variáveis explicativas adequadas não garantem que haja sobreposição da distribuição dos indivíduos tratados com os de controle. A adoção do método de pareamento pelo vizinho mais próximo, que foi utilizada no presente trabalho e cujo uso é o mais frequente na literatura, contorna esse problema, uma vez que compara cada indivíduo com o contrafactual que apresentar o escore de propensão mais próximo. No entanto, caso o contrafactual mais 
próximo esteja muito distante, o efeito estimado para o tratamento será enviesado. Por esse motivo, embora a redução do viés médio e a significância da maioria das variáveis explicativas do pareamento sejam indícios da qualidade do procedimento, é fundamental analisar a tabela de médias antes e após o pareamento, e a redução de viés, dados apresentados na Tabela 3.

Tabela 3 - Testes de médias entre produtores atendidos e não atendidos por assistência técnica antes e depois do pareamento

\begin{tabular}{|c|c|c|c|c|c|}
\hline \multirow{2}{*}{ Variável } & \multirow{2}{*}{ Amostra } & \multicolumn{2}{|c|}{ Média } & \multirow{2}{*}{ \% viés reduzido } & \multirow{2}{*}{$p>|t|$} \\
\hline & & Trat. & Cont. & & \\
\hline \multirow{2}{*}{ Idade } & Não pareada & 50,092 & 50,197 & \multirow{2}{*}{$-761,0$} & 0,859 \\
\hline & Pareada & 50,092 & 50,998 & & 0,196 \\
\hline \multirow{2}{*}{ utilPRONAF } & Não pareada & 0,34167 & 0,06494 & \multirow{2}{*}{93,4} & 0,000 \\
\hline & Pareada & 0,34167 & 0,32333 & & 0,501 \\
\hline \multirow{2}{*}{ Estudo } & Não pareada & 5,7767 & 3,722 & \multirow{2}{*}{96,2} & 0,000 \\
\hline & Pareada & 5,7767 & 5,6983 & & 0,722 \\
\hline \multirow{2}{*}{ FEM } & Não pareada & 0,065 & 0,08751 & \multirow{2}{*}{92,6} & 0,068 \\
\hline & Pareada & 0,065 & 0,06333 & & 0,906 \\
\hline \multirow{2}{*}{ Nbranco } & Não pareada & 0,34833 & 0,65584 & \multirow[b]{2}{*}{86,5} & 0,000 \\
\hline & Pareada & 0,34833 & 0,30667 & & 0,124 \\
\hline \multirow{2}{*}{ Cessionário } & Não pareada & 0,02667 & 0,06617 & \multirow{2}{*}{87,3} & 0,000 \\
\hline & Pareada & 0,02667 & 0,03167 & & 0,607 \\
\hline \multirow{2}{*}{ Posseiro } & Não pareada & 0,02833 & 0,04422 & \multirow{2}{*}{47,5} & 0,074 \\
\hline & Pareada & 0,02833 & 0,03667 & & 0,416 \\
\hline \multirow{2}{*}{ Arrendatário } & Não pareada & 0,03667 & 0,03711 & \multirow{2}{*}{$-659,2$} & 0,958 \\
\hline & Pareada & 0,03667 & 0,04 & & 0,764 \\
\hline Parceirn & Não pareada & 0,045 & 0,05473 & 315 & 0,330 \\
\hline Parcelro & Pareada & 0,045 & 0,03833 & 31,5 & 0,564 \\
\hline Outracondican & Não pareada & 0,00333 & 0,01453 & 851 & 0,025 \\
\hline Uutraconaicao & Pareada & 0,00333 & 0,00167 & 85,1 & 0,564 \\
\hline Prodsubsist & Não pareada & 0,09667 & 0,12523 & 65,0 & 0,049 \\
\hline Prodsubsist & Pareada & 0,09667 & 0,10667 & & 0,567 \\
\hline & Não pareada & 0,24833 & 0,25758 & & 0,634 \\
\hline Naoconsome & Pareada & 0,24833 & 0,245 & 63,9 & 0,894 \\
\hline & Não pareada & 0,055 & 0,0201 & & 0,000 \\
\hline lemempregtemp & Pareada & 0,055 & 0,04667 & 16,1 & 0,512 \\
\hline Tememnregnerm & Não pareada & 0,06167 & 0,02443 & & 0,000 \\
\hline remempregperm & Pareada & 0,06167 & 0,065 & 91,0 & 0,813 \\
\hline & Não pareada & 0,09167 & 0,26469 & & 0,000 \\
\hline Compconsdireto & Pareada & 0,09167 & 0,07167 & 88,4 & 0,206 \\
\hline & Não pareada & 0,42333 & 0,13575 & & 0,000 \\
\hline compempresa & Pareada & 0,42333 & 0,45333 & 89,6 & 0,295 \\
\hline & Não pareada & 0,13833 & 0,04762 & & 0,000 \\
\hline Compcooperativa & Pareada & 0,13833 & 0,135 & 96,3 & 0,867 \\
\hline Comnnronrietárin & Não pareada & 0,00833 & 0,00433 & 168 & 0,200 \\
\hline Compproprietarıo & Pareada & 0,00833 & 0,005 & 16,8 & 0,478 \\
\hline Comnintermediárin & Não pareada & 0,17333 & 0,28479 & 910 & 0,000 \\
\hline compintermediarıo & Pareada & 0,17333 & 0,16333 & 91,0 & 0,644 \\
\hline Outrocomnrador & Não pareada & 0,00333 & 0,00464 & 1555 & 0,659 \\
\hline Outrocomprador & Pareada & 0,00333 & 0,00667 & $-155,5$ & 0,413 \\
\hline & Não pareada & 0,16833 & 0,28386 & & 0,000 \\
\hline Norte & Pareada & 0,16833 & 0,16 & 92,8 & 0,697 \\
\hline & Não pareada & 0,13833 & 0,3859 & & 0,000 \\
\hline Nordeste & Pareada & 0,13926 & 0,14167 & 98,7 & 0,868 \\
\hline$C \Omega$ & Não pareada & 0,05 & 0,06988 & & 0,073 \\
\hline $\mathrm{CO}$ & Pareada & 0,05 & 0,04667 & 83,2 & 0,788 \\
\hline & Não pareada & 0,165 & 0,13946 & & 0,101 \\
\hline Sudeste & Pareada & 0,165 & 0,17167 & 73,9 & 0,758 \\
\hline
\end{tabular}

Fonte: Elaboração própria com base no modelo estimado. 
É possível observar na Tabela 3 que o pareamento resultou na redução de viés para a maioria das variáveis explicativas do modelo, com exceção das variáveis idade, a dummie para arrendatários e a dummie para outro comprador como principal comprador da produção. O aumento no viés dessas três variáveis, no entanto, não prejudica o pareamento, pois é irrelevante em termos absolutos, o que é atestado pelo fato de que, para todas as variáveis, a hipótese nula de que as médias dos grupos tratado e controle após o pareamento são iguais não pode ser rejeitada no nível de 10\% de significância.

A análise até aqui desenvolvida permite inferir que a construção dos grupos tratado e de controle é adequada aos requisitos da metodologia, permitindo a estimação não viesada ${ }^{6}$ dos efeitos da assistência técnica sobre a renda dos agricultores. Na Tabela 4, são apresentadas as estimações do efeito médio do tratamento (ATE) e do efeito médio do tratamento para os tratados (ATET).

Tabela 4 - Efeito médio da assistência técnica (ATE) e efeito médio da assistência técnica para os agricultores assistidos (ATET) sobre a renda mensal per capita na propriedade

\begin{tabular}{|c|c|c|c|c|c|c|}
\hline rendPerCapita & Coef. & Al Robust Std. Err. & $\mathbf{z}$ & $P>|z|$ & {$[95 \%$ Con } & Interval] \\
\hline $\begin{array}{c}\text { ATE } \\
\text { recebeuassistencia (1 vs 0) }\end{array}$ & 516,56 & 2,1932 & 235,53 & 0,000 & 512,27 & 520,86 \\
\hline $\begin{array}{c}\text { ATET } \\
\text { recebeuassistencia (1 vs 0) }\end{array}$ & 490,54 & 7,3549 & 66,70 & 0,000 & 476,13 & 504,96 \\
\hline
\end{tabular}

Fonte: Elaboração própria com base no modelo estimado.

Na Tabela 4, é possível observar que tanto o ATE quanto o ATET são positivos e estatisticamente significativos, comprovando o efeito positivo da assistência técnica sobre a renda dos agricultores familiares. Por meio da interpretação do ATE, pode-se afirmar que a utilização da assistência técnica no último trabalho desenvolvido na propriedade propiciou um aumento de, em média, $\mathrm{R} \$ 516,56$ na renda mensal dos agricultores familiares brasileiros no ano de 2014. Esse resultado é relevante quando se considera que, segundo os dados da PNAD, a renda mensal média dos agricultores familiares brasileiros em 2014 foi de $R \$ 2.071,297$.

O valor do ATET, por sua vez, indica que o acréscimo da renda mensal per capita para os agricultores que utilizaram assistência técnica no ano de 2014 , foi de $R \$ 490,54$, novamente confirmando o efeito positivo e estatisticamente significativo da assistência sobre a rentabilidade da propriedade. A diferença entre os valores do ATET e do ATE, no entanto, evidencia um aspecto extremamente relevante do problema de pesquisa: segundo o modelo estimado, no ano de 2014, a assistência para os agricultores não tratados teve um potencial de geração de renda superior àquele obtido pelos que utilizaram o serviço.

Comprovado o efeito positivo e estatisticamente significativo da assistência técnica sobre a renda dos agricultores, uma questão interessante seria avaliar o potencial de geração de renda deste serviço em termos agregados para os agricultores familiares brasileiros, resultado apresentado na Tabela 5.

Tabela 5 - Potencial de acréscimo de renda total mensal devido à utilização de assistência técnica, acréscimo efetivado e acréscimo não efetivado, para agricultores familiares em 2014.

\begin{tabular}{ccccc} 
& Total & Std. Err. & \multicolumn{2}{c}{ [95\% Conf. Interval] } \\
\hline Potencial total & $R \$ 1.127 .603 .050,00$ & $R \$ 121.009 .609,00$ & $R \$ 890.353 .664,00$ & $R \$ 1.364 .852 .440,00$ \\
Acrésc. efetivado & $R \$ 177.939 .694,00$ & $R \$ 69.121 .396,00$ & $R \$ 42.189 .955,00$ & $R \$ 313.689 .432,00$ \\
Acrésc. não efetivado & $R \$ 949.663 .362,00$ & $R \$ 99.358 .024,00$ & $R \$ 754.852 .280,00$ & $R \$ 1.144 .474 .440,00$ \\
\hline
\end{tabular}

Fonte: Elaboração própria com base no modelo estimado.

\footnotetext{
'É possível assumir que as estimações desenvolvidas após o pareamento não são viesadas, pois não foram omitidas variáveis relevantes, no pareamento, que não estejam altamente correlacionadas com as covariáveis incluídas. Isso garante, segundo Rosenbaum \& Rubin (1985), que o balanceamento seja consistente o suficiente para que os efeitos estimados do tratamento não sejam viesados.

${ }^{7} \mathrm{~A}$ estimativa da renda per capita mensal média para os agricultores familiares no ano de 2014 , considerando-se os pesos amostrais, foi de $R \$ 2.071,29$, variando entre $R \$ 1.992,87$ e $R \$ 2.149,72$ para um intervalo de confiança de $95 \%$.
} 
Os dados apresentados na Tabela 5 evidenciam que, conforme o modelo estimado, o aumento na renda mensal per capita dos agricultores familiares em 2014 devido ao efeito da assistência técnica foi bastante inferior ao potencial total estimado. O acréscimo de renda mensal estimado, caso todos os agricultores não assistidos em 2014 recebessem assistência, é de cerca de 949,66 milhões de reais, variando entre 754,85 milhões e 1,14 bilhão de reais, considerando-se o intervalo de confiança de 95\%. Esse valor representa $84,2 \%$ do potencial total de geração de renda devido à assistência técnica estimada pelo modelo, ou seja, a abrangência dos serviços de assistência técnica aos agricultores familiares no ano de 2014 limitou a geração de renda decorrente do serviço a menos de $16 \%$ do seu potencial. Para elucidar a relação entre a abrangência da assistência técnica e a geração de renda, é interessante analisar o percentual de agricultores familiares assistidos em 2014, dado apresentado na Tabela 6.

Tabela 6 - Percentual de agricultores que receberam assistência, para cada região do país

\begin{tabular}{|c|c|c|c|c|c|c|}
\hline Região do país & Norte & Nordeste & Centro-Oeste & Sudeste & Sul & Brasil \\
\hline $\begin{array}{l}\text { Percentual de agricultores } \\
\text { familiares assistidos em } \\
\text { cada região }\end{array}$ & $8,56 \%$ & $5,93 \%$ & $11,67 \%$ & $18,43 \%$ & $43,18 \%$ & $16,59 \%$ \\
\hline $\begin{array}{c}\text { População de agricultores } \\
\text { total estimada para cada } \\
\text { região* }\end{array}$ & 334.286 & 894.774 & 110.674 & 397.097 & 452.974 & 2.189 .805 \\
\hline
\end{tabular}

Fonte: Elaboração própria a partir de dados da PNAD (Instituto Brasileiro de Geografia e Estatística, 2017). * Apresenta a população total estimada de agricultores familiares por região, segundo o recorte amostral utilizado, incluindo aqueles que não receberam assistência técnica.

Na Tabela 6, é possível observar que 16,59\% dos agricultores familiares brasileiros utilizaram o serviço de assistência técnica em 2014, o que resultou em um acréscimo de renda, conforme apresentado na Tabela 5, de aproximadamente $15 \%$ do potencial total. Esses resultados comprovam aqueles observados na Tabela 4, segundo os quais o efeito da assistência em 2014 para os agricultores que não a receberam supera o acréscimo obtido pelos que foram assistidos. Em outras palavras, o aumento da abrangência da assistência técnica, mantido o padrão de qualidade do serviço prestado, não gera retornos marginais decrescentes.

Com isso, é possível inferir que, caso os custos da disponibilização do serviço de assistência técnica sejam inferiores ao acréscimo de renda estimado, é viável expandir o atendimento. Segundo dados disponíveis no Portal Transparência do Governo Federal, em 2014 o gasto do governo com o Programa de Assistência Técnica e Extensão Rural na Agricultura Familiar foi cerca de 17,5 milhões de reais (Brasil, 2014), um montante baixo quando se considera que a EMATER e outras agências do governo foram responsáveis pelo atendimento de $55,82 \%$ dos agricultores familiares assistidos no ano de $2014^{8}$. É importante considerar que, a partir dos anos 2000, a Política Nacional de Assistência Técnica e Extensão rural (PNATER) foi alicerçada em novas estratégias de descentralização dos serviços, em especial para agricultores familiares beneficiários de projetos de reforma agrária (Brasil, 2004, 2008). A descentralização preconizada pela nova política tem contemplado maior envolvimento de atores locais, em geral reunidos em arranjos cooperativos e selecionados pelo Estado através de editais públicos para a execução dos serviços de extensão rural, com contratos para atendimento a demandas específicas e por período determinado. Disto decorre que a presença do Estado pode ter importância efetiva mais expressiva do que o registrado (quase $56 \%$ de participação) porquanto efetivamente atuou como supervisor dos projetos de extensão rural contratados.

Considerando a representatividade da atuação estatal na prestação de assistência técnica, e a discrepância entre o gasto anual do governo (cerca de 17,5 milhões de reais) e o acréscimo de renda mensal resultante da assistência técnica estimado no presente trabalho

${ }^{8}$ Percentual estimado com base na PNAD de 2014, considerando-se o peso amostral. 
(R\$ 177.939.694,00), é possível inferir que a atuação estatal junto aos agricultores familiares foi lucrativa em termos do retorno agregado sobre a renda dos produtores. É importante considerar, no entanto, que o intervalo de confiança a 95\% para o acréscimo de renda mensal em 2014 foi bastante amplo, variando entre 42,18 e 313,68 milhões de reais, o que se deve à grande variância do efeito do tratamento estimado para os tratados. No entanto, mesmo diante da pouca precisão na estimação devido às limitações da base de dados, o efeito estimado da assistência técnica sobre a renda mensal dos produtores foi estatisticamente superior ao gasto anual do governo com as ações públicas de ATER.

Embora todas as estimações realizadas no presente trabalho estejam sujeitas às imprecisões características das análises estatística e econométrica, comuns a qualquer trabalho baseado no uso de dados oriundos de amostragem complexa, foram adotados os procedimentos mais adequados para minimizar possíveis erros e vieses, uma vez que as dificuldades metodológicas não inviabilizam uma análise de tal relevância, considerando-se a escassez de dados e trabalhos que abordam a problemática no nível econômico.

É possível concluir, nesse contexto, que os dados apresentados até então representam forte evidência de que a atuação governamental por meio de programas de assistência técnica e extensão rural à agricultores familiares apresenta retornos econômicos positivos e superiores ao gasto realizado. Além disso, é importante considerar que esse tipo de serviço apresenta benefícios em outras dimensões, que não podem ser estimados com base na análise da renda em curto prazo.

\section{Considerações finais}

O desenvolvimento de análises quantitativas dos efeitos da ATER é fundamental para o aperfeiçoamento da política. No presente trabalho, a aplicação do PSM permitiu a estimação, de forma metodologicamente consistente, do efeito da utilização de assistência técnica sobre a renda dos agricultores familiares brasileiros no ano de 2014 . Os resultados obtidos evidenciaram que a utilização de assistência técnica resultou em um acréscimo estatisticamente significativo na renda dos agricultores atendidos, o que evidencia a efetividade das ações de ATER enquanto instrumento de geração de renda.

Além disso, a análise em nível agregado permite inferir que o acréscimo de renda mensal total para os agricultores familiares no ano de 2014 devido às ações de ATER foi estatisticamente superior ao gasto anual do governo com o Programa de Assistência Técnica e Extensão Rural na Agricultura Familiar, um forte indício da viabilidade da expansão dos serviços prestados, que atenderam apenas cerca de $17 \%$ dos agricultores familiares no ano de 2014.

Conclui-se, por fim, que a política de ATER é um poderoso instrumento de geração de renda no Brasil, embora a abrangência das ações desenvolvidas no ano de 2014 sejam bastante limitadas. Ressalta-se também que a utilização de assistência técnica por agricultores familiares resulta em impactos em outras dimensões não analisadas no presente estudo. A expansão da política, associada à analises sistêmicas para seu constante aperfeiçoamento, é, portanto, um caminho promissor para o desenvolvimento econômico da agricultura familiar no Brasil.

\section{Referências bibliográficas}

Abadie, A., Angrist, J., \& Imbens, G. (2002). Instrumental variables estimates of the effect of subsidized training on the quantiles of trainee earnings. Econometrica, 70(1), 91-117.

Abramovay, R. (1998). Agricultura familiar e desenvolvimento territorial. Reforma Agrária, 28(1), 2.

Batalha, M. O., Buainain, A. M., \& Souza Filho, H. M. (2005). Tecnologia de gestão e agricultura familiar. Gestão Integrada da Agricultura Familiar. São Carlos: EdUFSCar.

Blundell, R., Dearden, L., \& Sianesi, B. (2005). Evaluating the effect of education on earnings: models, methods and results from the National Child Development Survey. Journal of the Royal Statistical Society. Series A, (Statistics in Society), 168(3), 473-512. 
Brasil. Ministério do Desenvolvimento Agrário. Secretaria de Agricultura Familiar. Departamento de Assistência Técnica e Extensão Rural. (2004). Política Nacional de Assistência Técnica e Extensão Rural. Brasília: MDA.

Brasil. Ministério do Desenvolvimento Agrário. Secretaria de Agricultura Familiar. Departamento de Assistência Técnica e Extensão Rural. (2008). Política Nacional de Assistência Técnica e Extensão Rural. Brasília: MDA.

Brasil. Governo Federal. Controladoria-Geral da União. (2014). Informações sobre transferência de recursos. Brasília: Portal da Transparência. Recuperado em 2 de março de 2018, de http://transparencia.gov.br/PortalTransparenciaGDProgramaPesquisaAcao.asp?ano=2014\&te xtoPesquisaPrograma=assistencia\%20tecnica\&codigoPrograma=1427

Brasil. (2017, maio 31). Decreto $n^{\circ} 9.064$, de 31 de maio de 2017. Dispõe sobre a Unidade Familiar de Produção Agrária, institui o Cadastro Nacional da Agricultura Familiar e regulamenta a Lei no 11.326, de 24 de julho de 2006, que estabelece as diretrizes para a formulação da Política Nacional da Agricultura Familiar e empreendimentos familiares rural. Diário Oficial [da] República Federativa do Brasil, Brasília. Recuperado em 1 de Março de 2018, de http://www.planalto.gov.br/ccivil_03/_ato2015-2018/2017/decreto/D9064.htm.

Brasil. Ministério da Agricultura Pecuária e Abastecimento - MAPA. (2018). Brasil. Brasília: MAPA. Recuperado em 2 de março de 2018, de http://www.agricultura.gov.br/noticias/agropecuariacresceu-13-em- 2017

Buainain, A. M., Romeiro, A. R., \& Guanziroli, C. (2003). Agricultura familiar e o novo mundo rural. Sociologias, 5(10), 312-347.

Caliendo, M., \& Kopeinig, S. (2008). Some practical guidance for the implementation of propensity score matching. Journal of Economic Surveys, 22(1), 31-72.

Caporal, F. R. (2008). Bases para uma Nova Ater Pública. Extensão Rural - Série de Estudos Rurais, v. 13, n. 1, 1-20.

Castro, C. N. (2015). Desafios da agricultura familiar: o caso da assistência técnica e extensão rural (Boletim Regional, Urbano e Ambiental, No. 12, pp. 49-59). Brasília: IPEA

Damasceno, N. P., Khan, A. S., \& Lima, P. V. P. S. (2011). O impacto do Pronaf sobre a sustentabilidade da agricultura familiar, geração de emprego e renda no Estado do Ceará. Revista de Economia e Sociologia Rural, 49(1), 129-156.

Dehejia, R. H., \& Wahba, S. (1999). Causal effects in nonexperimental studies: Reevaluating the evaluation of training programs. Journal of the American Statistical Association, 94(448), 1053-1062.

Dias, M. M. (2008). Políticas públicas de extensão rural e inovações conceituais: limites e potencialidades. Perspectivas em Políticas Públicas, 1(1), 101-114. Recuperado em 2 de fevereiro de 2018, de http://revista.uemg.br/index.php/revistappp/article/view/986/708

Feijó, R. L. C. (2001). The impact of a family farming credit program on the rural economy of Brazil. In Anais do XXIX Encontro nacional de Economia da ANPEC (Vol. 11, pp. 1-22). Niterói: ANPEC.

Gomes, A. P., Ervilha, G. T., Freitas, L. F., \& Nascif, C. (2016). A importância da assistência técnica para aumentar a eficiência e rentabilidade na produção de leite. In Anais do $54^{\circ}$ Congresso da Sociedade Brasileira de Economia, Administração e Sociologia Rural. Brasília: SOBER.

Guilhoto, J. J., Ichihara, S. M., Silveira, F. G. D., Diniz, B. P. C., Azzoni, C. R., \& Moreira, G. R. (2007). A importância da agricultura familiar no Brasil e em seus estados. Brasília: NEAD. Recuperado em 1 de fevereiro de 2017, de https://www.researchgate.net/profile/Carlos_Azzoni/publication/4731981_A_IMPORTANCIA_DA_AG RICULTURA_FAMILIAR_NO_BRASIL_E_EM_SEUS_ESTADOS/links/0fcfd 5089e9a9ec637000000.pdf

Hampf, A. C. (2013). Avaliação do impacto do PRONAF sobre a agricultura familiar no município de bonito, estado de Pernambuco, mediante o uso do propensity score matching (Dissertação de mestrado). Programa de Pós-Graduação em Administração e Desenvolvimento Rural da Universidade Federal Rural de Pernambuco, Recife.

Heckman, J. J., Ichimura, H., \& Todd, P. (1998). Matching as an econometric evaluation estimator. The Review of Economic Studies, 65(2), 261-294.

Instituto Brasileiro de Economia - IBRE. (2017). Comitê de datação de ciclos econômicos - CODACE. Rio de Janeiro: IBRE.

Instituto Brasileiro de Geografia e Estatística - IBGE. (2009). Censo Agropecuário 2006. Rio de Janeiro: IBGE. 
Instituto Brasileiro de Geografia e Estatística - IBGE (2017). Pesquisa Nacional por Amostras Domiciliares $P N A D$. Recuperado em 20 de Fevereiro de 2018, de https://www.ibge.gov.br/estatisticas/sociais/trabalho/9127-pesquisa-nacional-por-amostra-dedomicilios.html?=\&t=microdados.

Kageyama, A. (2003). Produtividade e Renda na Agricultura Familiar: Efeitos do PRONAF- Crédito. Agricultura, 50(2), 1-13.

Lalonde, R. J. (1986). Evaluating the econometric evaluations of training programs with experimental data. The American Economic Review, 76(4), 604-620.

Landau, E. C., da Cruz, R. K., Hirsch, A., Pimenta, F. M., \& Guimarães, D. P. (2012). Variação geográfica do tamanho dos módulos fiscais no Brasil. Minas Gerais: Embrapa Milho e Sorgo.

Magalhães, A. (2005). The family farm program in Brazil: the case of Parana. In Anais do Congresso da Sociedade Brasileira de Economia e Sociologia Rural (pp. 1-14). Ribeirão Preto: SOBER.

Menezes Filho, N., Peixoto, B., Pinto, C. C. X., Lima, L., Foguel, M. N., \& Barros, R. P. (2012). Avaliação econômica de projetos sociais. São Paulo: Dinâmica Gráfica e Editora, 2012.

Oliveira, M. M. (1999). As circunstâncias da criação da extensão rural no Brasil. Cadernos de Ciência \& Tecnologia, 16(2), 97-134.

Peixoto, M. (2008). Extensão rural no Brasil - uma abordagem histórica da legislação (pp. 1-50). Brasília: Núcleo de Estudos e pesquisas do Senado.

Pereira, S. (2004). Avaliação da Política de Agricultura Familiar: uma abordagem de efeito-fixo. In Anais do XVII Congresso da SOBER. Brasília: SOBER.

Quirino, T. R., Garagorry, F. L., \& Sousa, C. P. (2002). Diagnóstico sociotécnico da agropecuária brasileira: I. produtores (Documentos, 58 p.). Brasília: Embrapa Secretaria de Administração Estratégica.

Rosenbaum, P. R., \& Rubin, D. B. (1983). The Central role of the propensity score in observational studies for causal effects. Biometrika, 70(1), 41-55.

Rosenbaum, P. R., \& Rubin, D. B. (1985). Constructing a control group using multivariate matched sampling methods that incorporate the propensity score. The American Statistician, 39(1), 33-38.

Rosenbaum, P. R. (2002). Overt bias in observational studies. In P. R. Rosenbaum (Ed.), Observational studies (pp. 71-104). New York: Springer.

Rubin, D. B. (1973). The use of matched sampling and regression adjustment to remove bias in observational studies. Biometrics, 29, 185-203.

Rubin, D. B. (1979). Using multivariate matched sampling and regression adjustment to control bias in observational studies. Journal of the American Statistical Association, 74(366a), 318-328.

Ruttan, V. W., \& Hayami, Y. (1984). Toward a theory of induced institutional innovation. The Journal of Development Studies, 20(4), 203-223.

Schultz, T. W. (1965). A transformação da agricultura tradicional. Rio de Janeiro: Zahar.

Sianesi, B. (2004). An evaluation of the Swedish system of active labor market programs in the 1990s. The Review of Economics and Statistics, 86(1), 133-155.

Silva, R. P. (2013). As especificidades da nova ATER para Agricultura Familiar. Revista NERA, 16, 150-166.

Smith, J. A., \& Todd, P. E. (2005). Does matching overcome LaLonde's critique of nonexperimental estimators? Journal of Econometrics, 125(1-2), 305-353.

Wanderley, M. N. B. (2013). Agricultura familiar e campesinato: rupturas e continuidade. Estudos Sociedade e Agricultura, 1, 42-61.

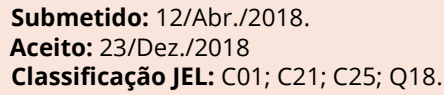

